\title{
Dynamic Behavior Analysis of Non-Contacting Hydrodynamic Finger Seal Based on Fluid-Solid-Interaction Method
}

\author{
Hua Su${ }^{1}$, and Jiru Wang ${ }^{2}$ \\ 1 School of Mechanical Engineering, Northwestern Polytechnical University, 710072 Xi'an, China \\ 2 School of Electrical Engineering, Xi'an Jiaotong University, 710049, Xi'an, China
}

\begin{abstract}
Finger seal is an advanced compliant seal and can be utilized to separate high (HP) and low pressure (LP) zones in high speed rotating shaft environment. The work to be presented concerns the dynamic behavior of a repetitive section of a two-layer finger seal with high- and padded low-pressure laminates. The dynamic performance of the finger seal are analyzed by the coupled fluid-solid-interaction (FSI) simulations. By using the commercial software ANSYS-CFX, the numerical simulation results of interactions between the gas flow and fingers structural deformation are described when the radial periodic excitation from the shaft applies to the finger seal. And the gas film loading capacity, gas film stiffness and leakage varied with time are put forward in different working conditions. Compared with the dynamic performance analysis results based on equivalent dynamic method, the FSI dynamic analysis shows some different characteristics which are more accordance with actual circumstance. Moreover, it is shown that under low pressure differential and high rotation speed the non-contacting finger seal with advance features both in sealing effectiveness and potential unlimited life span can be obtained by rational structure design. But for the non-contacting finger seal with circumferential convergent pad working in high pressure and low rotating speed conditions, it is difficult to improve the sealing performance by the way of changing the structure parameters of finger seal. It is because the high pressure plays a major role on this sealing situation.
\end{abstract}

\section{Introduction}

Finger seal is new type seal presented after labyrinth seal and brush seal which is regarded as a revolutionary new technology in air-to-air sealing for secondary flow control and gas path sealing in gas turbine engines. It is given more attention in past two decades because of its excited performances and relative low manufacture costs. The first generation finger seal is a kind of contacting seal, which is composed of multiple staggered laminates with each having a large number of flexible fingers around the rotor surface[1]. It is found that the finger seal has demonstrated leakage considerably lower than that shown by conventional labyrinth seal, and at a cost much lower than that of the commonly used brush seal. However, when the radial run-out of rotor exist unavoidably, the flexible finger feet may contact with the rotor surface and result in heating and wear especially in high-speed and high-temperature operation conditions. Therefore, non-contacting finger seal has been came up with in recent years. The main significant structural feature of non-contacting finger seal is aerodynamic selfacting lift pads added at the bottom of the low pressure fingers. And certain clearance is reserved between the pads and the rotor surface. Under the differential pressure and rotary motion of rotor, hydrodynamic pressure will generate in the flow film between rotor and pads to lift the finger seal and maintain the non-contacting operation condition. This non-contacting finger seal are expected to be used in high-speed and high-temperature sealing conditions with better performance.

The researches on non-contacting finger seal at present mainly include the following fields: firstly, the different structural design of lifting pad including circumferential converged[2], axial and circumferential double converged[3], step-shaped, herringbone grooved[4] and micro-textured [5]etc. Secondly, analysis on static and dynamic performances of finger seal under different working conditions. Non-contacting finger seal is generally serviced in dynamic condition, so it is crucial to concern about its dynamic performance and working stability. Most current analysis on dynamic performance of finger seal is based on mass-spring-damper equivalent dynamic model. Though it can get the tacking characteristics of seal to the stimulus from rotor and vibration performance of seal, there are still some disparities from practice due to some assumptions applied in the equivalent model. In order to further understand the dynamic performance of the hydrodynamic finger seal and the flow characteristic of the fluid, the fluid-solid coupling analysis method based on the prototype of the sealing system is used in presented work to concern about the dynamic performance of the non-contacting finger seal. 
This paper introduces the seal geometry in second part. And the fluid-solid coupling analysis method is discussed in third part. Then the dynamic performances of non-contacting finger seal in different working conditions and with different structures are analyzed in the fourth part, and it ends with the conclusions.

\section{Finger seal geometry}

The finger seal with circumferential converged pad consists of front plate, spacer, high-pressure (HP) finger, low-pressure (LP) finger with pad and back plate, as shown in Figure 1. The finger stick profile is traced by means of arc. The detailed geometry parameters in Figure 1 are shown in Table 1.

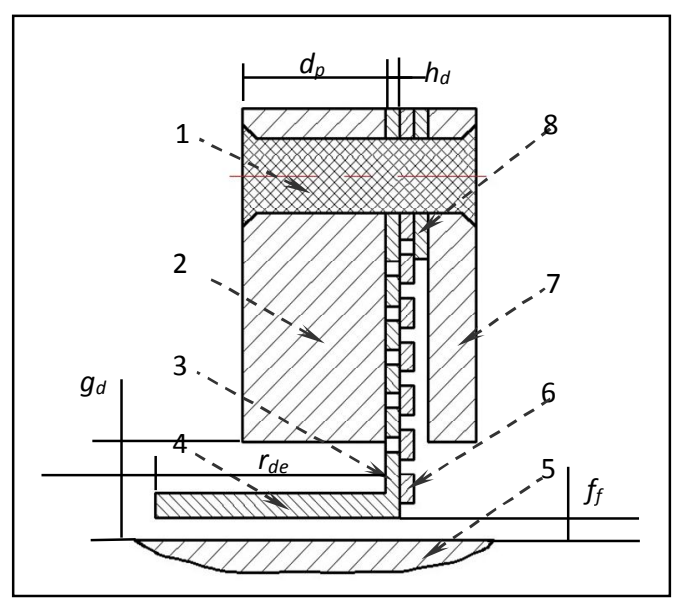

1-vivet 2-back plate 3-LP finger 4-pad 5-rotor 6-HP finger 7-front plate 8-spacer

(a) Axial cross section

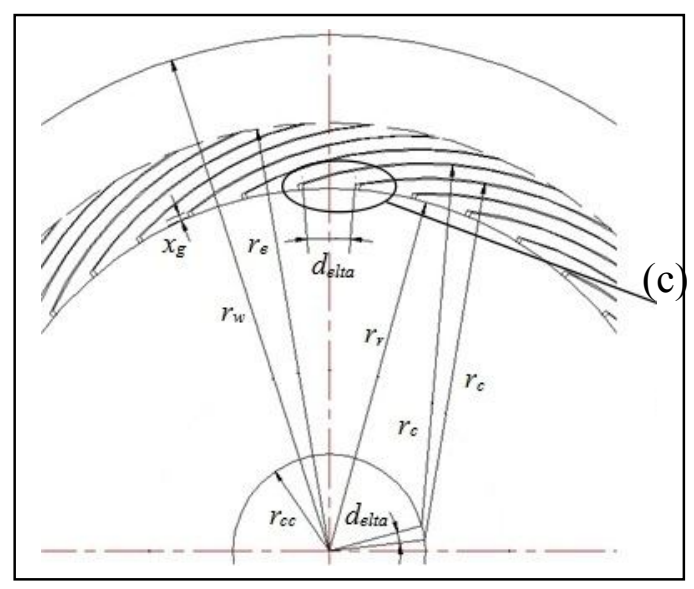

(b) Head face

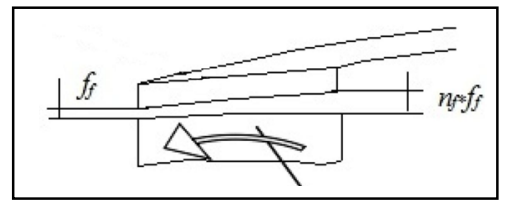

(c) Partial enlarged view

Figure 1. Geometry of finger seal with circumferential converged pad
Table 1. Geometric Values of the Basic Finger Seal

\begin{tabular}{|l|c|c|}
\hline Finger Geometry & Symbol & Value \\
\hline Outer radius of finger $(\mathrm{mm})$ & $\mathrm{r}_{\mathrm{w}}$ & 122.65 \\
\hline Radius of finger root $(\mathrm{mm})$ & $\mathrm{r}_{\mathrm{e}}$ & 116.45 \\
\hline Rotor Radius $(\mathrm{mm})$ & $\mathrm{r}_{\mathrm{r}}$ & 108 \\
\hline Stick Arc Radius $(\mathrm{mm})$ & $\mathrm{r}_{\mathrm{c}}$ & 114.6 \\
\hline Radius of Center Circle $(\mathrm{mm})$ & $\mathrm{r}_{\mathrm{cc}}$ & 20 \\
\hline Finger Repeat Angle $\left.{ }^{\circ}\right)$ & $\mathrm{d}_{\mathrm{elta}}$ & 4.444 \\
\hline Radial height of HP foot & $\mathrm{x}_{\mathrm{g}}$ & 1.22 \\
\hline Minimum radial clearance $(\mathrm{mm})$ & $\mathrm{f}_{\mathrm{f}}$ & 0.00635 \\
\hline wedge ratio of clearance & $\mathrm{n}_{\mathrm{f}}$ & 3 \\
\hline Axial width of LP pad $(\mathrm{mm})$ & $\mathrm{r}_{\mathrm{de}}$ & 6.35 \\
\hline Radial gap of the back plate $(\mathrm{mm})$ & $\mathrm{g}_{\mathrm{d}}$ & 1 \\
\hline Thickness of back plate $(\mathrm{mm})$ & $\mathrm{d}_{\mathrm{p}}$ & 5 \\
\hline Thickness of finger seal $(\mathrm{mm})$ & $\mathrm{h}_{\mathrm{d}}$ & 0.762 \\
\hline
\end{tabular}

\section{Fluid-solid interaction analysis on dynamic behaviors of finger seal}

The dynamic behavior of non-contacting finger seal is analyzed based on the displacement response of finger pad to the rotor stimulus in given working conditions. The rotor produce radial run-out because of its unbalanced mass, which will indirectly impact on pad by gas film and results in radial movement of the fingers. Since the high compliance of the fingers, the force from the gas film move the finger seal and results in a change of flow field profile. These in turn contribute to further change the load distribution and flowing condition of gas film. Thus the dynamic analysis of non-contacting finger seal is based on transient fluid-structure interaction(FSI) analysis of finger seal.

\subsection{Finite element model of fluid-solid field}

Considering the circumferential convergence finger seal is a cyclic symmetry structure, to simplify the dynamic analysis procedure, a circular unit including a LP finger stick and a two-half HP finger stick are chosen to establish the solid structure model (shown in Figure 2), and the corresponding flow field between the finger pad and rotor is as gas flow field model (shown in figure 3 ). Here the solid and gas film models are established based on commercial software ANSYS-CFX.

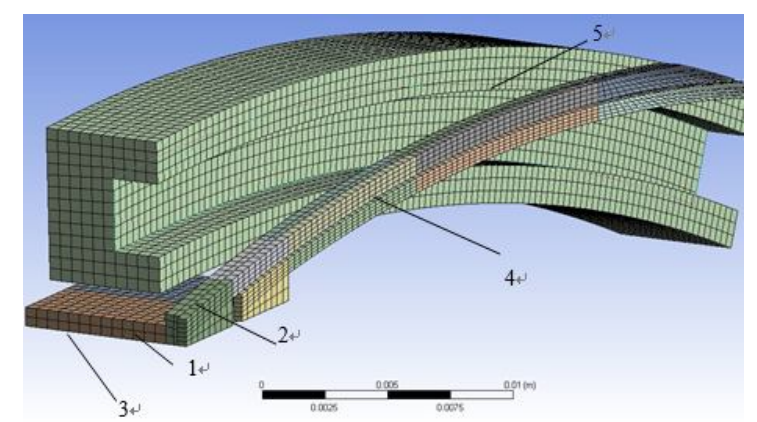

1-half HP finger 2-half HP finge 3-hydrodynamic pad 4-back plate 5-LP finger

Figure 2. Solid model of non-contacting finger seal 


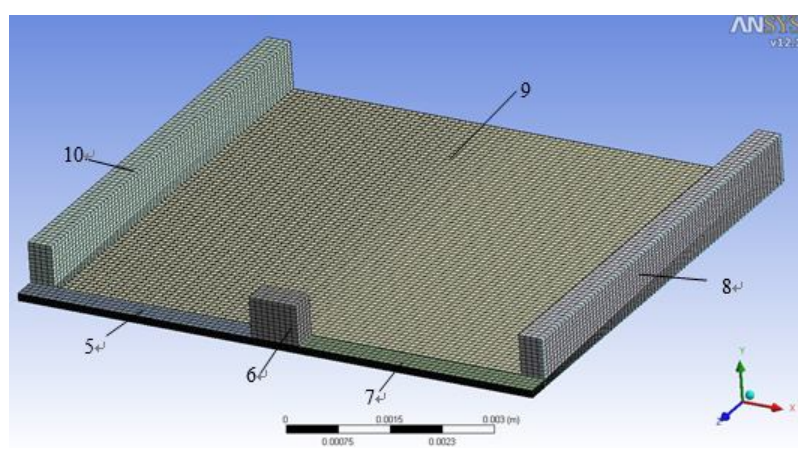

Figure 3. Gas film model of non-contacting finger seal

\subsection{Boundary conditions}

The mechanical boundary conditions in solid model of circumferential convergence finger seal on dynamic analysis including:

1)The outer faces of HP and LP are fixed as well as the back plate.2)The surface in HP finger face to high pressure is applied to pressure differential.3) The surface under the pad is set to be fluid-solid intersection surface.

4) The contact surface between LP plate and back plate is set to be friction contact-pair and the friction coefficient $\mu$ is 0.2. 5) The contact surface between HP and LP is set to be friction contact-pair to demonstrate the relative movement between HP and LP slice. Because there is only dynamic pad in LP stick which results in different lift movement between HP and LP.

According to Figure 3, the boundary conditions in fluid flow filed are as follows:1) The inlet (front surfaces 5, 6 and 7) are set opening boundaries and the high pressure are given. 2) Top surfaces 8 and 10 and the back surface 9 are outlet of the gas field, which are set opening boundaries and the low pressure are given.3) The two cut sides of 8 and 10 are set to be cyclical boundaries.4) The lower surface of 9 is contacting with rotor surface, which is set moving wall and the velocity and dynamic stimulus are given.5) The upper surface of 9 is interface of solid and fluid, which is set to be wall.

\subsection{Forms of stimulus}

The unbalanced force of rotor is a harmonic exciting force. Considering the phase and frequency of the radial run-out of rotor are same as those of the unbalanced force of rotor, the rotor displacement stimulus may substitute for unbalanced force of the rotor, and the amplitude the displacement stimulus is the radial runout of the rotor. Then the rotor displacement stimulus can be expressed as

$$
y(t)=\alpha \sin \left(\frac{n \pi}{30} t\right)
$$

Where, $\alpha$ is the runout of the rotor, $n$ is the rotate speed of the rotor.

\subsection{Parameters of dynamic performance}

Here we use leakage M, film load capacity F, and gas film stiffness $\mathrm{K}$ to describe the dynamic behavior of the non-contacting finger seal. A finger seal with lower leakage, higher load capacity and higher stiffness simultaneously exhibits good dynamic performances.

\subsubsection{Leakage}

The seal leakage is relative with the area and velocity of the flow at outlet. These two factors are all vary with time. The mass flow leakage of the seal at a moment can be calculated by the following formula

$$
M=\int \rho \cdot v d s
$$

Where, $\int \mathrm{ds}$ is the area of outlet, $v$ is the velocity of the gas in outlet, $\rho$ is the density of the gas.

The average leakage of the seal in a rotor rotating cycle is defined as

$$
M_{\mathrm{av}}=\frac{\sum_{i=1}^{n} M}{n}
$$

The variable coefficient of the leakage at every moment is defined as

$$
M_{\mathrm{cv}}=\frac{M-M_{\mathrm{av}}}{M_{\mathrm{av}}}
$$

\subsubsection{Load capacity}

The load capacity of the gas film of non-contacting seal is the radial film force applied to the finger pad. The load force is calculated as

$$
F=\left|\int P d A\right|
$$

Where, $\int \mathrm{dA}$ is the area of the coupling surface, $P$ is the pressure at the coupling surface.

\subsubsection{Gas film stiffness}

Gas film stiffness is the derivative of film load with respect to film thickness, which is defined as

$$
K=-\frac{d F}{d \delta}
$$

Where, $F$ is film loading force, $\delta$ is film thickness. In a periodic excitation the film thickness of finger seal can be calculated approximately as

$$
\delta=\bar{Y}(t)-y(t)
$$

Where, $\bar{Y}(t)$ is average position of the pad bottom, $\mathrm{y}(\mathrm{t})$ is the rotor displacement.

\subsection{Calculating method}

Based on SST model and K- $\omega$ equations the finger seal flow is simulated as turbulent model. The convergence is set as $10^{-4}$. The original position of rotor and finger are set according to the installation. In a periodic motion of rotor the stimulation is discretized to compute the fluid flow and solid deformation at a moment, and its result is set as the initial value for the next compute point till the end of the whole computing period. 


\section{Results and discussing}

\subsection{Compared with the results from equivalent dynamic model}

Using the structural and working conditions from Reference[6],the finger seal displacement responses are calculated by the FSI method from the current paper, and the results are compared with the method of equivalent dynamic model from reference[6]. The comparing results are shown in Figure 4.

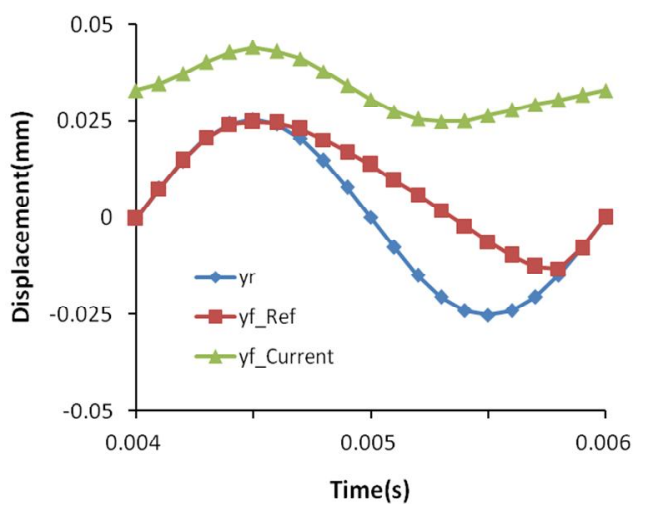

Figure 4. Finger's displacement response of the two methods

From Figure 4 it is found that the two results are quite different. Firstly, in Reference[6] the initial gap is set to the initial minimum film thickness, $6.35 \mu \mathrm{m}$, then the initial displacement response is zero because the initial minimum film alteration due to the hydrodynamic effect is not considered. In current computation the initial film thickness will increase to about $30 \mu \mathrm{m}$ at pressure of $6.9 \mathrm{KPa}$ and rotating speed of $30000 \mathrm{rpm}$. Secondly, in the first $1 / 4$ increasing period, or the equivalent dynamic model, the finger followed the rotor closely because the initial film thickness is very small with greater film stiffness. And the finger's curvature is not considered in the equivalent dynamic model, so the finger stick stiffness is constant. However, in current paper the finger response is a little slower because the initial film thickness is bigger and the film thickness is decrease gradually hence to the film stiffness increase subsequently. And at the same time the finger's curvature is decreased resulting the increasing of finger's stiffness. Thirdly, in the period from the highest position to the lowest position, the finger's response lags behind the rotor because of the hysteresis of finger seal. Though this phenomenon can be seen in the two results, but the displacement decreasing value for dynamic model is greater than that of the current method because the film stiffness in the equivalent dynamic model is bigger. Lastly, in the last $1 / 4$ period the finger's rising position is different. For dynamic model when the rotor rise from the lowest position the fingers move down due to the inertia. But actually when the film stiffness is small and the finger stiffness is bigger, the finger will stop at its lowest position due to the frictional hysteresis before the rotor arrives at the lowest position. And the finger will rise up following the rotor due to the increasing film load capacity.

\subsection{Effect of pad parameters on the finger seal dynamic performance}

The load capacity of dynamic finger seal is mainly caused by the dynamic pad. Here the varieties of dynamic pad parameters are discussed on the performance of finger seal.

\subsubsection{Wedge ratio of clearance}

Figure 5 and Figure 6 show the different wedge ratio effects on the leakage and film stiffness respectively.

For the case of lower pressure $(1 \mathrm{psi}, 6.9 \mathrm{kPa})$-higher velocity (30krpm), when the minimum film thickness is given the clearance of the film increase with the wedge ratio, as well as enhance the dynamic effect which increase the film load capacity. So it is found that Mav is increasing and $M \mathrm{cv}$ is decreasing. Meanwhile the enlarged film thickness will decrease the film stiffness especially in the condition of lower pressure drop and higher velocity, which is shown in Figure 6. Hence in low pressure and high velocity working conditions the wedge ratio should be selected not only to avoid the contact between the rotor and finger pad but also to maintain lower leakage.

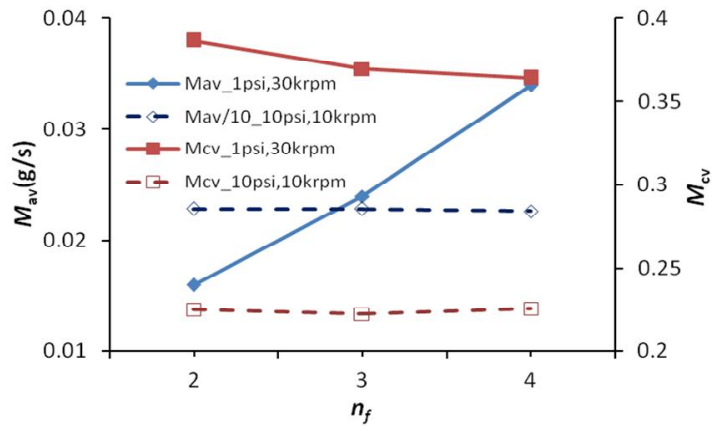

Figure 5. Effects of wedge ration on leakage

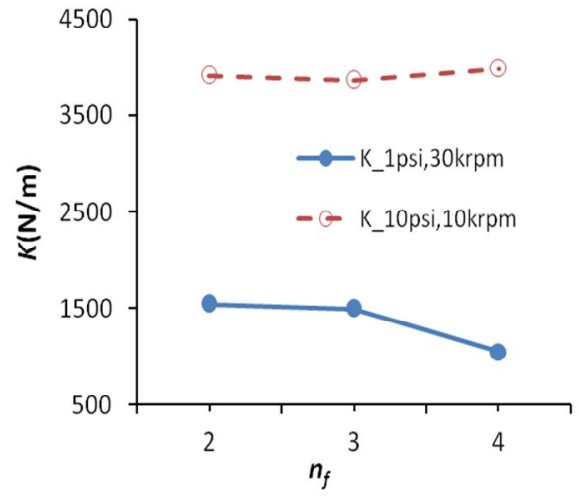

Figure 6. Effects of wedge ration on film stiffness

For the case of high pressure $(10 \mathrm{psi}, 69 \mathrm{kPa})$-lower velocity(10krpm), the leakage and film stiffness do not vary obviously. Because the pad lifting displacement 
$(80 \mu \mathrm{m})$ is much greater than the original clearance $(10 \mu \mathrm{m})$ which make the film clearance increase largely. Then the effect of the variation of the film due to the wedge ratio is relatively small. In higher pressure conditions the film form have little effect to the finger seal performance. So in later parts we just discuss the performance in lower pressure -higher velocity conditions.

\subsubsection{Minimum film thickness}

Figure 7 and Figure 8 show the leakage and film stiffness with different minimum film thickness with 1 psi pressure drop and $30 \mathrm{krpm}$ velocity. When the wedge ratio of film is keep constant, the film thickness will enlarge with the minimum film thickness increase, which will result in increasing of leakage and fluid velocity. Then the load capacity is enhanced. However with the film thickness increasing the film stiffness will decrease. So for lower pressure-higher velocity conditions, thinner film thickness can keep high film stiffness and low leakage.

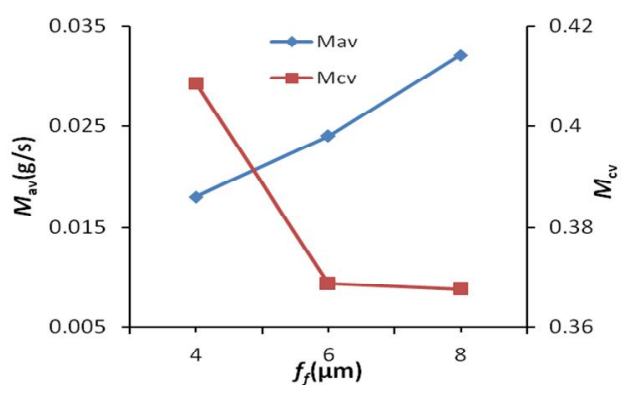

Figure 7. Effects of minnmum film thickness on leakage (1psi,30krpm)

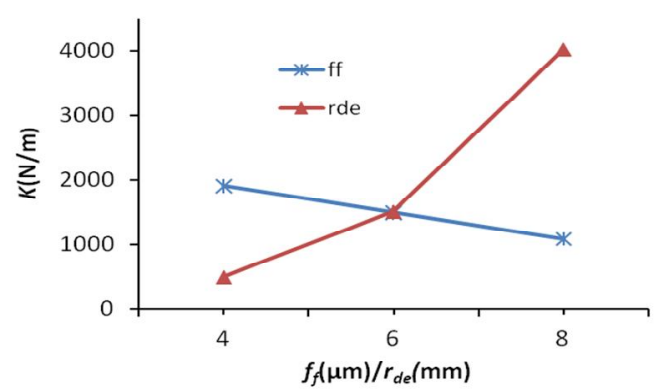

Figure 8. Effects of minnmum film thickness and axial length of pad on film stiffness(1psi,30krpm)

\subsubsection{Axial length of the dynamic pad}

Figure 9 and Figure 8 show the effects of axial length of pad on the leakage and film stiffness respectively. When the axial length of pad increase the fluid flow resistance increase, then the leakage decrease and the film stiffness increase. The pad can follow the rotor well when rotor has radial runout and the leakage variation is small too. For lower pressure-high velocity case it is better to lengthen the pad axial length to keep high film stiffness and low leakage.

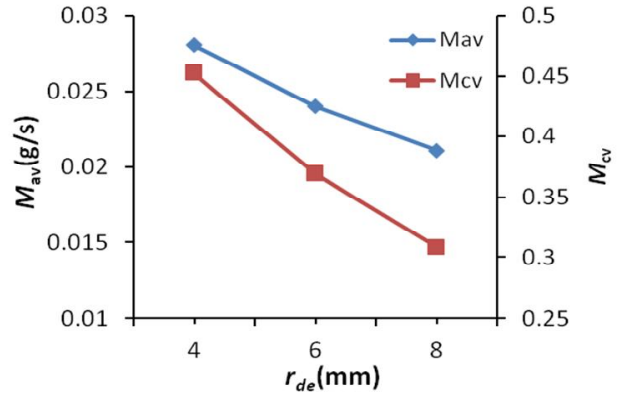

Figure 9. Effects of axial length of pad on leakage(1psi,30krpm)

\section{Conclusions}

The dynamic performance of the non-contacting finger seal is analyzed based on 3D FSI method, which can describe the movement between the rotor and dynamic pad much accurately. However, it cost more computing time than solving the equivalent dynamic model.

It is found when the non-contacting finger seal working in higher pressure drop the pad will lift obviously, which results in high leakage and the dynamic potential may not be developed. It is necessary to improve the performance of the finger seal adapting to high pressure conditions.

For lower pressure and high velocity conditions the pad parameters will influence the leakage and film stiffness of the seal definitely. For the cases studied in this paper, when the wedge ratio is 3 , the minimum film thickness is 6 to $7 \mu \mathrm{m}$, and the longer pad axial length, the film stiffness keep higher and leakage is lower as well as the pad can avoid contact with the rotor, which maintain better dynamic performance.

\section{Acknowledgment}

The authors would like to extend their appreciation to the National Natural Science Foundation of China for supporting this work under Grant 51575445.

\section{References}

1. G K. Arora, M.P.Proctor, B. M.Steinetz. and I. R.Delgado, AIAA-99-2686, 1-18(1999)

2. M.J. Braun, V.V. Kudriavtsev, B.M. Steinetz, and M.P. Proctor, The 9th International Symposium on Transport Phenomena and Dynamics of Rotating Machinery, Honolulu, Hawaii, February 10-14, 18( 2002)

3. H. Marie, 42nd AIAA/ASME/SAE/ASEE Joint Propulsion Conference \& Exhibit 9-12 July 2006, Sacramento, California. AIAA 2006-4931.118(2006).

4. D.P. Fleming,"Gas Seal Pad With HerringboneGrooved Rotor-Stiffness and Load Capacity,"NASA/TM-2006-214333,pp.1-13 (2006)

5. D.X Lang, H. Su, Acta Aeronautica et Astronatica Sinica, 33, 1540-1546(2012)

6. M.J.Braun , H.M Pierson., D.Deng, Tribology Transactions, 48, 531-547(2005) 